\title{
Diets and coexistence of the sea urchins Lytechinus variegatus and Arbacia punctulata (Echinodermata) along the central Florida gulf coast
}

\author{
Janessa Cobb, John M. Lawrence* \\ Department of Biology, University of South Florida, Tampa, Florida 33620, USA
}

\begin{abstract}
The basis for coexistence of similar species is fundamental in community ecology. One mechanism for coexistence is differentiation of diets. Lytechinus variegatus and Arbacia punctulata coexist in different microhabitats along the Florida gulf coast. Their great difference in morphology might affect their choice of microhabitats and diet. We analyzed diets of both species at 1 offshore and 1 nearshore site where both occurred in relatively equal numbers, an offshore site dominated by A. punctulata and an offshore site dominated by L. variegatus. Gut contents were analyzed to determine the diet. A. punctulata prim. consumed sessile invertebrates except on dates when algal availability was higher than normal. L. variegatus primarily consumed macroflora except on dates when macroflora was extremely limited. Electivity indices revealed no strong preferences for particular species of algae, although $L$. variegatus consumed many drift species. A. punctulata and $L$. variegatus both fed in a random manner, although they avoided particular species of algae known to contain high concentrations of secondary metabolites. The diet of $A$. punctulata was correlated with algae only over rubble outcroppings at the offshore site with the highest biomass. Diets of offshore populations were more similar to each other, regardless of the presence of conspecifics, than to those of populations at Caspersen Beach (nearshore site). As diets do not overlap, distribution of individuals at a location would not be affected by interspecific competition for food. However, intraspecific competition may be high due to low site productivity.
\end{abstract}

KEY WORDS: Sea urchins - Arbacia punctulata $\cdot$ Lytechinus variegatus $\cdot$ Diet $\cdot$ Gulf of Mexico · Coexistence Resale or republication not permitted without written consent of the publisher

\section{INTRODUCTION}

Tokeshi (1999, p. 1) noted that species coexistence is 'beguilingly simple and, yet, is a remarkably accurate description of the natural world' and that 'the theme of coexistence of species is central in community ecology'. Pairs of sea urchin species coexist in numerous habitats. They may belong to different genera, e.g. Paracentrotus lividus (Lamarck) and Arbacia lixula (Linnaeus) in the Mediterranean (Boudouresque \& Verlaque 2001) and Loxechinus albus (Molina) and Tetrapygus niger L. Agassiz (Vásquez 2001) along the Chilean coast or be of the same genus with distinct morphologies, e.g. Strongylocentrotus purpuratus (Stimpson) and Strongylocentrotus franciscanus
(Agassiz) (Tegner 2001). Typically species found in pairs differ in microhabitats and diets.

The sea urchins Lytechinus variegatus (Lamarck) and Arbacia punctulata (Lamarck) occur throughout the Gulf of Mexico and the Caribbean in a variety of environments (Hendler et al. 1995). Along the central Florida gulf coast they usually occupy distinct microhabitats, L. variegatus on sand and A. punctulata on rock (Hill \& Lawrence 2003). Although the diet of L. variegatus in seagrass beds has been well documented, few studies have recorded the diet at depths greater than $5 \mathrm{~m}$. In shallow water, it is a generalist grazer of fresh and detrital algae, seagrasses (Moore et al. 1963, Lowe \& Lawrence 1976, Vadas et al. 1982, Keller 1983, Greenway 1995, Couto 1998, Beddingfield 
\& McClintock 1999), and occasionally animals (Lowe \& Lawrence 1976). The diet of A. punctulata is not well documented. Parker (1932) and Harvey (1956) stated that it is an omnivore, and Karlson (1975) called it a generalized carnivore. A. dufresnei is primarily carnivorous in Argentinian waters (Penchaszadeh \& Lawrence 1999). The Mediterranean A. lixula feeds on algae and invertebrates (Frantzis et al. 1988), although studies on interaction between Paracentrotus lividus and A. lixula have focused primarily on herbivory (Benedetti-Cecchi \& Cinelli 1995, Benedetti-Cecchi et al. 1998, Bulleri et al. 1999, 2002). Understanding the feeding habits of sea urchins is essential to assess the impact of these animals within a community and their interactions with each other and their prey.

Very few studies document the feeding habits of Lytechinus variegatus and Arbacia punctulata outside seagrass beds. However, $L$. variegatus and $A$. punctulata coexist at many offshore locations with highly variable algal communities in the Gulf of Mexico (Cobb \& Lawrence 2003, Hill \& Lawrence 2003). Therefore, it is reasonable to assume that the diets and interactions of $L$. variegatus and A. punctulata will also vary between these populations. In this study, we asked (1) how the diets of $L$. variegatus and A. punctulata overlap, (2) if diets differ when the species coexist and when only 1 species dominates a site, (3) if each species selects specific foods or feeds randomly, and (4) if each species shows a preference for feeding over sand or rubble.

\section{MATERIALS AND METHODS}

Field observations and collection. Three offshore sites and 1 nearshore site along the central west coast of Florida (Fig. 1) were selected based on a priori knowledge of sea urchin populations. Observations were made and specimens collected at all sites using SCUBA. Collection dates, GPS coordinates, depths, and water temperature are given in Table 1. Site 1, approximately $37.5 \mathrm{~km}$ offshore, was dominated by Arbacia punctulata, although individuals of Lytechinus variegatus were found occasionally. The substratum consisted primarily of flat, rubble outcroppings of limestone with sparse patches of sand. Macroalgae were evenly distributed and included large stands of Sargassum sp. several meters in length. Sessile invertebrates were common and primarily included sponges and ascidians. Site 1 is $15.1 \mathrm{~km}$ west of Sites 2 and 3.

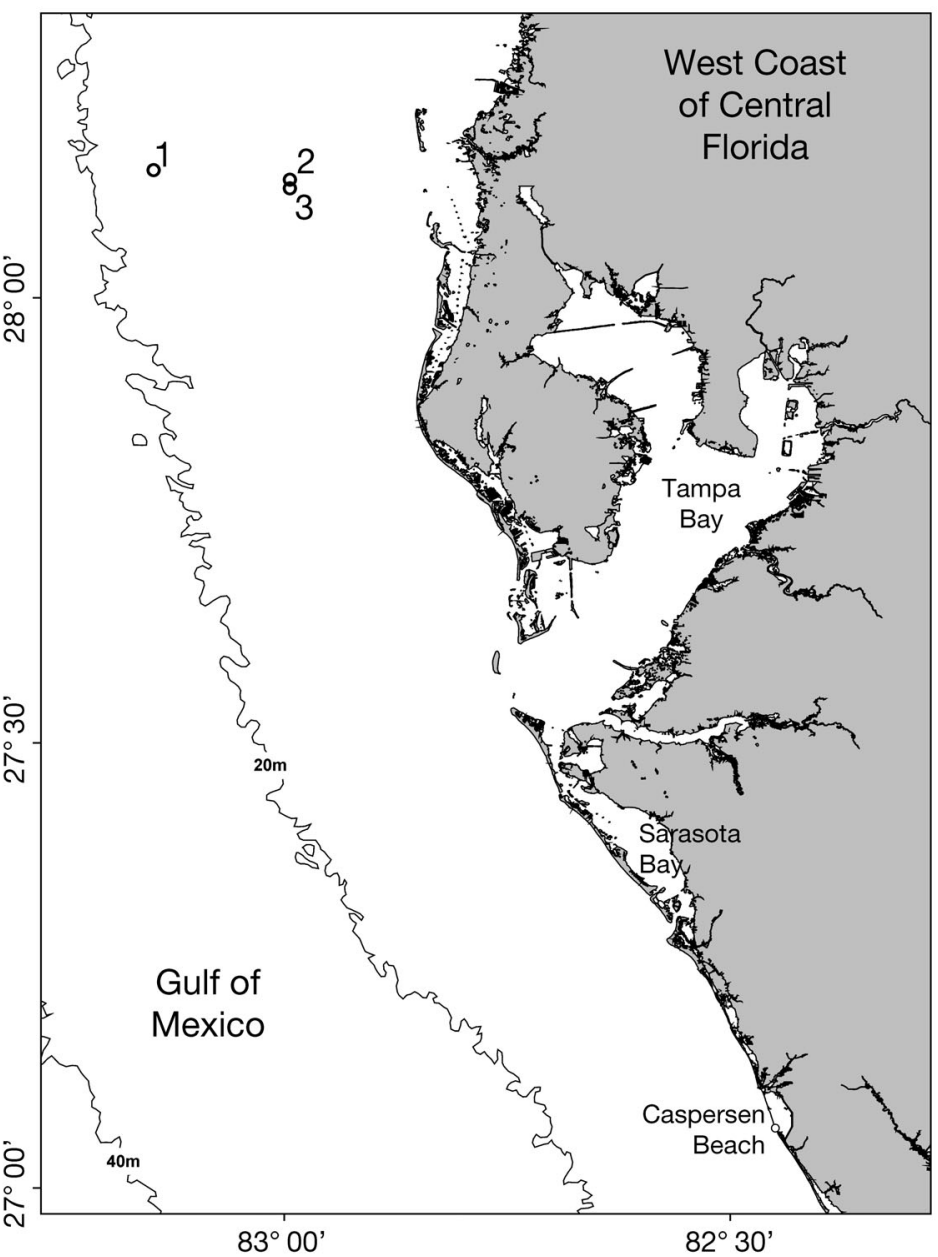

Fig. 1. Locations of offshore (1-3) and near-shore (Caspersen Beach) sites on the central Florida gulf coast shelf

At Site 2, both Lytechinus variegatus and Arbacia punctulata were present in large numbers. Large limestone outcroppings were moderately scattered over swaths of sand. $L$. variegatus was often found on sand, heaped with various shells or algae, while A. punctulata was only found attached to rubble. Sponges, ascidians, and some macroalgae were found attached to rubble outcroppings. Site 2 is only $193 \mathrm{~m}$ from Site 3.

Only Lytechinus variegatus was present at Site 3, which consisted primarily of barren sand. A few natural outcroppings were sparsely distributed and smaller in size than those at Site 2. A few concrete bricks acted as an artificial reef substratum for some ascidians and other sessile invertebrates.

The nearshore site is approximately $300 \mathrm{~m}$ off Caspersen Beach. Both species of sea urchins were found in similar numbers. Large rubble outcroppings were abundant on sand flats at approximately $6 \mathrm{~m}$ in depth. Arbacia punctulata was only present on rubble outcroppings while Lytechinus variegatus was found on 
Table 1. Sampling locations, depths and dates in the Gulf of Mexico. Water temperature is also shown at sampling sites. Water temperature was lowest at Caspersen Beach on January 17, 2000. Dash indicates not measured

\begin{tabular}{|lcccc|}
\hline & Site 1 & Site 2 & Site 3 & Caspersen \\
\hline Location & $28^{\circ} 8.56^{\prime} \mathrm{N}$ & $28^{\circ} 7.56^{\prime} \mathrm{N}$ & $28^{\circ} 7.45^{\prime} \mathrm{N}$ & $27^{\circ} 4^{\prime} \mathrm{N}$ \\
& $83^{\circ} 8.82^{\prime} \mathrm{W}$ & $82^{\circ} 59.70^{\prime} \mathrm{W}$ & $82^{\circ} 59.70^{\prime} \mathrm{W}$ & $82^{\circ} 27^{\prime} \mathrm{W}$ \\
Depth (m) & 17.7 & 11.9 & 11.9 & 5.8 \\
& \multicolumn{4}{c}{ Water temperature $\left({ }^{\circ} \mathrm{C}\right)$} \\
\hline Sampling date & \multicolumn{5}{c}{} \\
Sep 29, 1999 & - & - & - & 27.2 \\
Oct 2, 1999 & 26.7 & 26.7 & 26.7 & - \\
Jan 17, 2000 & - & - & - & 17.2 \\
Apr 8, 2000 & 21.7 & 21.7 & 23.3 & - \\
Jun 15, 2000 & - & - & - & 29.4 \\
Aug 23, 2000 & - & - & - & 30.0 \\
Nov 12, 2000 & 22.2 & 21.7 & 21.7 & - \\
\hline
\end{tabular}

Differences in size and repletion indices between sites were determined by ANOVA and Tukey's multiple comparison test.

Gut content analysis. Gut contents were analyzed using the point-count method described by Jones (1968) to determine abundance of each item in the gut as done by Verlaque (1984) and Frantzis et al. (1988). Gut contents were sieved through a $595 \mu \mathrm{m}$ sieve to remove fine sediment and items too small to identify. Then $2 \mathrm{ml}$ of the remaining gut contents were placed into a petri dish with a $2.5 \times$ $2.5 \mathrm{~cm}$ grid. Gridlines were spaced in increments of $25 \mathrm{~mm}$ resulting in 121 intersections. The frequency with

both substratum types. Caspersen Beach closely resembles Site 2, although rubble outcroppings merge into sand patches, while microhabitats at Site 2 are discrete. Limestone outcroppings supported a number of species of ascidians, sponges, hydroids, and gorgonians. Macroalgae were sparsely distributed over rubble at sampling depths. Drift algae were common over sand at most depths, and large stands of red macroalgae and Caulerpa spp. were noted in areas of high hydrodynamics at approximately $3.5 \mathrm{~m}$ in depth.

Ten specimens of the dominant species of sea urchin (or both species if each was equally abundant) were collected from each site for gut content analysis. Each sea urchin was injected with a $4 \%$ formalin solution (Vadas 1985) to preserve gut contents. Sea urchins of similar size were collected haphazardly from both sand and rubble. Samples were collected from all offshore sites in the fall of 1999, and spring and fall of 2000. During the spring of 2000, an algal bloom prevented divers from locating rubble outcroppings to collect samples over hard substrata at 1 offshore site. Nearshore collections at Caspersen Beach were made in the fall of 1999, and winter, spring, and summer of 2000.

Samples of macroalgae and plants (collectively referred to as flora) were collected from quadrats over rubble and sand, along with the sea urchins, to document the species present within each habitat and to estimate available biomass at each site. These data are published in Cobb \& Lawrence (2003).

Measurements of sea urchin size and calculation of repletion index. Test diameter was measured and wet weight obtained for each sea urchin prior to dissection. A repletion index was calculated according to Régis (1980) and Verlaque \& Nédelec (1983):

(wet weight of gut contents $\times 100$ ) / wet body weight which each specimen in the gut contacted the intersections of the grid was recorded and used to determine the relative abundance of each food type within the gut. Five replicates were analyzed for each sea urchin to calculate average abundance and to insure consistency.

Plant and macroalgal specimens in the gut were identified as with field specimens. All samples were separated by species and identified by external morphology and examination of cross sections according to Dawes (1974) with taxonomic names corrected using Wynne (1986). In some cases, gut specimens were indistinguishable and had to be grouped into broader categories. For example, species of the genera Micropeuce, Polysiphonia, Murrayella, and Lophosiphonia were counted under the group name 'Family Rhodomelaceae (4).' Other members of the family Rhodomelaceae distinguishable from these 4 genera were counted separately. To avoid confusion and simplify quantification, fragments of material covered by several species of minute epiphytes were grouped together as 'epiphytes'.

Animals in the gut were identified to the genus level whenever possible. Due to the difficulty in accurately identifying partially digested fragments, most animals were grouped into higher taxa. References used to identify most animals were Sterrer (1986) and Ruppert \& Fox (1988). Hydroids could often be classified to genus according to Fraser (1944). Some bryozoans were identifiable according to Shier (1964); however, most cheilostomes and cyclostomes could not be classified beyond order. Fragments of crustaceans other than barnacles were often too small to identify species. Some tunicates could be classified according to Van Name (1945) and Plough (1978). Animals were grouped into phyla for statistical analysis. 
The total number of grid intersections using the point-count method for all animal and floral material consumed by each sea urchin were averaged for each site and collection date. Flora and fauna consumed by each species of sea urchin were compared using a paired Student's $t$-test or the Wilcoxin signed rank test.

The contingency coefficient $(C)$ was used to measure the degree of association between the diet of sea urchins and different sites and sampling dates:

$$
C=\sqrt{\chi^{2} /\left(N+\chi^{2}\right)}
$$

The frequency of each food was used to calculate chisquare $\left(\chi^{2}\right)$, and the sum of all observed frequencies $(N)$ was used to compute $C$. Values of $C$ were also used to measure the similarity of the diets of coexisting sea urchins at Site 2 and Caspersen Beach.

The Index of Proportional Similarity (Sc) of Czekanowski (as used in Verlaque \& Nédelec 1983) was used to compare floral diet with floral availability of each microhabitat within a site to determine if sea urchins were feeding over specific substrates:

$$
\mathrm{Sc}=\sum \operatorname{MIN}\left(P_{i}, Q_{i}\right) / 100
$$

Variables $P_{i}$ and $Q_{i}$ represent percentage abundance of food item $i$ in groups $P$ and $Q$, which in this case is food $i$ consumed by one sea-urchin species and availability of food $i$ in the environment. The Index of Proportional Similarity is the sum of the minimum (MIN, the lower of the 2 values $P_{i}$ and $Q_{i}$ ) percentage abundance of each food item, divided by 100 . This index was used to compare flora consumed by each sea urchin species with available flora over rubble, sand, and rubble and sand combined. Flora over sand was also compared with flora over rubble to calculate differences between microhabitats at each site.

Abundance of each floral species consumed by Arbacia punctulata and Lytechinus variegatus was compared with availability of floral material at each site using Strauss's Food Selectivity Index $(L)$ to determine random or selective feeding of each food by sea urchins (Strauss 1979):

$$
L=r_{i}-p_{i}
$$

where $r_{i}$ is relative abundance of food $i$ in the gut and $p_{i}$ is relative abundance of food $i$ in the environment. Therefore Strauss's index ranges from -1.0 (avoidance) to +1.0 (preference). Values were categorized as follows:

\begin{tabular}{rrr}
\multicolumn{3}{c}{ Index range } \\
\hline-1.00 & to & -0.75 \\
-0.74 & to & -0.25 \\
-0.24 & to & 0.24 \\
0.25 & to & 0.74 \\
0.75 & to & 1.00
\end{tabular}

Selectivity

Strong avoidance

Moderate avoidance Random feeding

Moderate preference Strong preference

\section{RESULTS}

\section{Size and repletion index}

Size of Arbacia punctulata did not vary greatly between collection dates at most sites (Table 2). Repletion indices were greater at Site 1 than at either Site 2 or Caspersen Beach.

Lytechinus variegatus was significantly larger at both Sites 2 and 3, and showed much less variation among sampling dates than individuals at Caspersen Beach (Table 2). Repletion indices fluctuated considerably between collection dates although no distinction between sites was detected.

\section{Diet composition}

Laurencia spp. (covered with epiphytes, and other small epiphytic red algae of the Rhodomelaceae) were the most frequently consumed macroflora by Arbacia punctulata at Site 1 (Table 3). A. punctulata primarily ingested small epiphytic algae or drift algae at all other sites. Lytechinus variegatus frequently consumed a greater diversity of macroflora than A. punctulata. Both sea urchins consumed a wide diversity of sessile invertebrates at all locations, particularly hydrozoans, ectoprocts, and barnacles (Table 4).

Table 2. Arbacia punctulata and Lytechinus variegatus. Test diameter $(\mathrm{mm})$ and repletion indices at offshore and nearshore sites in the Gulf of Mexico. (mean $\pm \mathrm{SD}_{i} \mathrm{n} / \mathrm{p}$ :

\begin{tabular}{|c|c|c|c|c|}
\hline & \multicolumn{2}{|c|}{ Arbacia punctulata } & \multicolumn{2}{|c|}{ Lytechinus variegatus } \\
\hline & $\begin{array}{c}\text { Test } \\
\text { diameter }\end{array}$ & $\begin{array}{l}\text { Repletion } \\
\text { index }\end{array}$ & $\begin{array}{c}\text { Test } \\
\text { diameter }\end{array}$ & $\begin{array}{l}\text { Repletion } \\
\text { index }\end{array}$ \\
\hline \multicolumn{5}{|l|}{ Site 1} \\
\hline Oct 99 & $39.1 \pm 4.1$ & $5.5 \pm 1.3$ & $\mathrm{n} / \mathrm{p}$ & $\mathrm{n} / \mathrm{p}$ \\
\hline Apr 00 & $33.9 \pm 6.7$ & $9.7 \pm 1.6$ & $\mathrm{n} / \mathrm{p}$ & $\mathrm{n} / \mathrm{p}$ \\
\hline Nov 00 & $40.8 \pm 4.6$ & $7.3 \pm 1.9$ & $\mathrm{n} / \mathrm{p}$ & $\mathrm{n} / \mathrm{p}$ \\
\hline \multicolumn{5}{|l|}{ Site 2} \\
\hline Oct 99 & $30.4 \pm 4.2$ & $4.4 \pm 2.7$ & $73.1 \pm 2.7$ & $6.8 \pm 1.8$ \\
\hline Apr 00 & $30.9 \pm 4.3$ & $5.2 \pm 2.1$ & $74.7 \pm 2.1$ & $11.5 \pm 3.1$ \\
\hline Nov 00 & $36.3 \pm 3.2$ & $4.9 \pm 1.7$ & $71.7 \pm 2.9$ & $7.5 \pm 2.0$ \\
\hline \multicolumn{5}{|l|}{ Site 3} \\
\hline Oct 99 & $\mathrm{n} / \mathrm{p}$ & $\mathrm{n} / \mathrm{p}$ & $73.6 \pm 2.9$ & $8.3 \pm 1.8$ \\
\hline Apr 00 & $\mathrm{n} / \mathrm{p}$ & $\mathrm{n} / \mathrm{p}$ & $73.0 \pm 3.7$ & $13.2 \pm 3.5$ \\
\hline Nov 00 & $\mathrm{n} / \mathrm{p}$ & $\mathrm{n} / \mathrm{p}$ & $73.8 \pm 2.4$ & $6.0 \pm 1.2$ \\
\hline \multicolumn{5}{|c|}{ Caspersen } \\
\hline Sep 99 & $27.9 \pm 4.1$ & $2.5 \pm 0.8$ & $46.9 \pm 3.9$ & $7.1 \pm 3.3$ \\
\hline Jan 00 & $\mathrm{n} / \mathrm{a}$ & $\mathrm{n} / \mathrm{a}$ & $58.6 \pm 2.8$ & $10.1 \pm 3.1$ \\
\hline Jun 00 & $30.7 \pm 5.1$ & $3.7 \pm 2.5$ & $57.3 \pm 3.8$ & $7.4 \pm 2.3$ \\
\hline Aug 00 & $38.3 \pm 6.5$ & $4.5 \pm 1.3$ & $62.7 \pm 7.0$ & $5.1 \pm 1.8$ \\
\hline
\end{tabular}
species not present; $\mathrm{n} / \mathrm{a}$ : data not available; $\mathrm{n}=7$ for $L$. variegatus in August 2000; $\mathrm{n}=10$ on all other collection dates) 
Table 3. Arbacia punctulata and Lytechinus variegatus. Frequency of ingested flora (n = 10). 1: Site 1, 10/2/99 (mo/d/yr); 2: Site 1, 4/8/00; 3: Site 1, 11/12/00; 4: Site 2, 10/2/99; 5: Site 2, 4/8/00; 6: Site 2, 11/12/00; 7: Site 3, 10/2/99; 8: Site 3, 4/8/00; 9: Site 3, 11/12/00; 10: Caspersen, 9/29/99; 11: Caspersen, 1/17/00; 12: Caspersen, 6/15/00; 13: Caspersen, 8/23/00

\begin{tabular}{|c|c|c|c|c|c|c|c|c|c|c|c|c|c|c|c|c|c|c|c|}
\hline \multirow[t]{2}{*}{ Collection } & \multicolumn{9}{|c|}{ Arbacia punctulata } & \multicolumn{10}{|c|}{ Lytechinus variegatus } \\
\hline & 1 & 2 & 3 & 4 & 5 & 6 & 10 & 12 & 13 & 4 & 5 & 6 & 7 & 8 & 9 & 10 & 11 & 12 & 13 \\
\hline Cyanophyta & 1 & 1 & 0 & 1 & 0 & 0 & 0 & 4 & 2 & 0 & 0 & 0 & 0 & 0 & 3 & 0 & 0 & 0 & 1 \\
\hline Chrysophyta & 0 & 0 & 0 & 0 & 0 & 0 & 0 & 0 & 3 & 1 & 0 & 0 & 1 & 0 & 0 & 0 & 0 & 0 & 1 \\
\hline \multicolumn{20}{|l|}{ Chlorophyta } \\
\hline Bryopsis pennata & 0 & 0 & 0 & 0 & 0 & 0 & 0 & 0 & 0 & 0 & 5 & 0 & 1 & 7 & 0 & 0 & 0 & 0 & 0 \\
\hline Caulerpa spp. & 0 & 0 & 0 & 0 & 0 & 0 & 1 & 0 & 3 & 0 & 1 & 0 & 7 & 4 & 0 & 0 & 0 & 0 & 3 \\
\hline Cladophora spp. & 0 & 5 & 0 & 4 & 7 & 0 & 1 & 1 & 2 & 1 & 10 & 0 & 0 & 9 & 3 & 0 & 0 & 0 & 1 \\
\hline Codium spp. & 0 & 0 & 0 & 0 & 0 & 0 & 0 & 0 & 0 & 1 & 1 & 0 & 0 & 0 & 0 & 1 & 0 & 0 & 0 \\
\hline Enteromorpha spp. & 0 & 0 & 0 & 0 & 0 & 0 & 0 & 0 & 0 & 0 & 0 & 0 & 0 & 0 & 0 & 0 & 0 & 1 & 0 \\
\hline Pseudocodium floridanum & 0 & 0 & 0 & 0 & 0 & 0 & 0 & 0 & 0 & 1 & 0 & 0 & 0 & 0 & 0 & 0 & 0 & 2 & 0 \\
\hline Udotea spp. & 2 & 1 & 2 & 0 & 0 & 0 & 0 & 0 & 0 & 0 & 2 & 0 & 2 & 1 & 1 & 0 & 0 & 0 & 0 \\
\hline \multicolumn{20}{|l|}{ Phaeophyta } \\
\hline Cladosiphon occidentalis & 0 & 4 & 0 & 0 & 0 & 0 & 0 & 0 & 0 & 0 & 3 & 0 & 0 & 6 & 0 & 0 & 0 & 0 & 0 \\
\hline Dictyota spp. & 5 & 4 & 1 & 2 & 1 & 0 & 0 & 0 & 3 & 10 & 3 & 1 & 7 & 1 & 1 & 0 & 1 & 0 & 6 \\
\hline Lobophora variegata & 0 & 0 & 0 & 1 & 2 & 1 & 0 & 0 & 0 & 0 & 0 & 1 & 0 & 0 & 2 & 0 & 0 & 0 & 0 \\
\hline Sargassum spp. & 0 & 0 & 0 & 0 & 0 & 0 & 0 & 0 & 0 & 0 & 8 & 4 & 0 & 8 & 6 & 0 & 1 & 0 & 0 \\
\hline Sphacelaria tribuloides & 0 & 0 & 0 & 0 & 1 & 3 & 0 & 0 & 0 & 2 & 6 & 3 & 0 & 4 & 3 & 0 & 0 & 0 & 0 \\
\hline \multicolumn{20}{|l|}{$\begin{array}{l}\text { Rhodophyta } \\
\text { Acanthophora muscoides }\end{array}$} \\
\hline Agardhiella spp. & 0 & 0 & 0 & 0 & 0 & 0 & 1 & 0 & 2 & 0 & 0 & 0 & 1 & 2 & 1 & 6 & 7 & 9 & 4 \\
\hline Amphiroa spp. & 0 & 1 & 0 & 0 & 0 & 0 & 0 & 0 & 0 & 0 & 0 & 0 & 0 & 0 & 0 & 0 & 0 & 0 & 0 \\
\hline Batophora oerstedi & 0 & 1 & 0 & 0 & 0 & 0 & 0 & 0 & 0 & 0 & 0 & 0 & 0 & 0 & 0 & 0 & 0 & 0 & 0 \\
\hline Botryocladia occidentalis & 3 & 1 & 0 & 0 & 0 & 0 & 0 & 0 & 0 & 0 & 2 & 0 & 0 & 0 & 0 & 9 & 1 & 0 & 0 \\
\hline Caloglossa leprieuri & 0 & 0 & 0 & 0 & 0 & 0 & 0 & 0 & 0 & 0 & 0 & 0 & 0 & 0 & 0 & 0 & 1 & 0 & 0 \\
\hline Centroceras spp. & 0 & 0 & 0 & 0 & 0 & 0 & 0 & 0 & 0 & 0 & 1 & 0 & 0 & 0 & 0 & 0 & 0 & 1 & 2 \\
\hline Ceramium spp. & 0 & 4 & 0 & 0 & 5 & 0 & 0 & 7 & 6 & 1 & 10 & 0 & 0 & 8 & 0 & 1 & 0 & 0 & 5 \\
\hline Champia parvula & 0 & 2 & 0 & 0 & 0 & 1 & 0 & 0 & 1 & 1 & 4 & 0 & 0 & 5 & 0 & 0 & 0 & 1 & 2 \\
\hline Chondria spp. & 0 & 2 & 1 & 0 & 0 & 0 & 0 & 0 & 0 & 0 & 2 & 0 & 0 & 1 & 0 & 0 & 2 & 0 & 0 \\
\hline Dasya spp. & 0 & 0 & 1 & 0 & 0 & 0 & 0 & 0 & 0 & 0 & 0 & 0 & 0 & 0 & 0 & 0 & 0 & 0 & 0 \\
\hline Euchema nudum & 0 & 0 & 0 & 0 & 0 & 0 & 0 & 0 & 0 & 0 & 5 & 0 & 0 & 0 & 0 & 2 & 0 & 2 & 1 \\
\hline Family Rhodomelaceae (4) & 3 & 7 & 0 & 0 & 1 & 0 & 0 & 7 & 4 & 5 & 7 & 0 & 2 & 6 & 1 & 0 & 1 & 8 & 1 \\
\hline Gelidium spp. & 0 & 0 & 0 & 1 & 1 & 2 & 0 & 0 & 0 & 1 & 0 & 0 & 0 & 1 & 1 & 1 & 1 & 0 & 4 \\
\hline Gracilaria spp. & 0 & 0 & 1 & 0 & 1 & 0 & 0 & 0 & 1 & 0 & 1 & 0 & 3 & 0 & 1 & 0 & 4 & 10 & 5 \\
\hline Нурпеа spp. & 0 & 0 & 0 & 0 & 0 & 0 & 1 & 3 & 7 & 0 & 1 & 0 & 0 & 0 & 0 & 7 & 7 & 10 & 6 \\
\hline Jania spp. & 0 & 0 & 1 & 0 & 0 & 0 & 0 & 0 & 0 & 0 & 2 & 0 & 0 & 1 & 0 & 0 & 0 & 0 & 0 \\
\hline Kylinia crassipes & 0 & 0 & 0 & 0 & 0 & 0 & 1 & 0 & 0 & 0 & 10 & 1 & 0 & 9 & 2 & 0 & 0 & 0 & 0 \\
\hline Laurencia spp. & 6 & 8 & 5 & 2 & 1 & 0 & 0 & 0 & 0 & 1 & 1 & 2 & 0 & 0 & 1 & 2 & 0 & 0 & 0 \\
\hline Lithothamnion spp. & 0 & 0 & 8 & 1 & 4 & 2 & 0 & 0 & 0 & 5 & 4 & 7 & 2 & 3 & 7 & 0 & 0 & 0 & 0 \\
\hline Spyridia filamentosa & 0 & 0 & 0 & 0 & 0 & 0 & 0 & 0 & 0 & 0 & 0 & 0 & 0 & 0 & 0 & 0 & 1 & 2 & 1 \\
\hline Epiphytes & 0 & 8 & 8 & 0 & 0 & 0 & 0 & 0 & 0 & 3 & 0 & 1 & 0 & 0 & 0 & 0 & 0 & 0 & 0 \\
\hline Unidentifiable algae & 5 & 6 & 5 & 0 & 1 & 0 & 2 & 5 & 8 & 3 & 9 & 2 & 4 & 6 & 7 & 4 & 5 & 5 & 7 \\
\hline Anthophyta & & & & & & & & & & & & & & & & & & & \\
\hline Thalassia testudinum & 1 & 0 & 0 & 0 & 0 & 0 & 0 & 0 & 0 & 7 & 4 & 10 & 4 & 3 & 6 & 0 & 1 & 1 & 0 \\
\hline Unidentifiable plant & 0 & 0 & 0 & 0 & 0 & 0 & 0 & 0 & 0 & 0 & 3 & 1 & 2 & 1 & 2 & 0 & 2 & 1 & 0 \\
\hline
\end{tabular}

Comparison of the amount of flora and fauna in the gut contents of Arbacia punctulata showed that all populations normally ingested more animals than plants or ingested both in equal proportions (Fig. 2). However, Lytechinus variegatus consumed significantly more flora than fauna during the spring and summer at all sites (Fig. 3). Both species consumed significantly more animals than flora in November 2000 at all offshore locations and during October 1999 at Site 3.

Diets of different populations within species are compared in Table 5A. The diet of Arbacia punctulata at Site 1 during October 1999 was significantly different from the diet of populations at all other sites. The diet of $A$. punctulata at Site 1 was also significantly different from specimens at Caspersen Beach on all collection dates. Lytechinus variegatus at Caspersen Beach had very little overlap in diet between dates and was significantly different from all offshore collections.

The diet of Arbacia punctulata and Lytechinus variegatus was also compared at sites where these species coexist (Table 5B). The diets of coexisting sea urchins 
Table 4. Arbacia punctulata and Lytechinus variegatus. Frequency of ingested fauna $(\mathrm{n}=10)$. 1: Site 1, 10/2/99 (mo/d/yr); 2: Site 1, 4/8/00; 3: Site 1, 11/12/00; 4: Site 2, 10/2/99; 5: Site 2, 4/8/00; 6: Site 2, 11/12/00; 7: Site 3, 10/2/99; 8: Site 3, 4/8/00; 9: Site 3, 11/12/00; 10: Caspersen, 9/29/99; 11: Caspersen, 1/17/00; 12: Caspersen, 6/15/00; 13: Caspersen, 8/23/00. See Cobb (2002) for a more detailed list of animals consumed by sea urchins

\begin{tabular}{|c|c|c|c|c|c|c|c|c|c|c|c|c|c|c|c|c|c|c|c|}
\hline \multirow[t]{2}{*}{ Collection } & \multicolumn{9}{|c|}{ Arbacia punctulata } & \multicolumn{10}{|c|}{ Lytechinus variegatus } \\
\hline & 1 & 2 & 3 & 4 & 5 & 6 & & & & 4 & 5 & & 7 & 8 & 9 & & & & 13 \\
\hline Porifera & 3 & 3 & 7 & 1 & 0 & 6 & 4 & 1 & 5 & 2 & 3 & 3 & 1 & 0 & 1 & 2 & 4 & 0 & 2 \\
\hline nidaria & & 5 & 6 & 4 & 0 & 2 & 4 & 3 & 5 & 10 & 4 & 2 & 6 & 5 & 10 & 6 & 7 & 3 & \\
\hline ctoprocta & 7 & 6 & 5 & 3 & 8 & 7 & 0 & 0 & 1 & 6 & 8 & 10 & 5 & 6 & 9 & 0 & 5 & 1 & 0 \\
\hline ipuncula & 0 & 0 & 0 & 0 & 0 & 0 & 0 & 0 & 0 & 0 & 0 & 0 & 0 & 0 & 1 & 0 & 0 & 0 & 0 \\
\hline Mollusc & 0 & 0 & 1 & 1 & 0 & 1 & 1 & 1 & 0 & 0 & 0 & 0 & 0 & 0 & 0 & 0 & 0 & 1 & 1 \\
\hline Annelida & 0 & 0 & 0 & 0 & 0 & 0 & 0 & 1 & 0 & 0 & 0 & 0 & 0 & 0 & 0 & 1 & 0 & 1 & 0 \\
\hline \multicolumn{20}{|l|}{ Arthropoda } \\
\hline Balanus sp. & 0 & 0 & 0 & 7 & 0 & 10 & 9 & 10 & 7 & 10 & 4 & 10 & 10 & 9 & 10 & 3 & 2 & 10 & 6 \\
\hline Errar & 0 & 1 & 0 & 0 & 0 & 0 & 2 & 1 & 1 & 7 & 3 & 7 & 3 & 0 & 8 & 2 & 1 & 1 & 2 \\
\hline Chordata & 5 & 1 & 7 & 3 & 1 & 10 & 0 & 0 & 0 & 7 & 4 & 9 & 5 & 1 & 7 & 0 & 0 & 0 & 0 \\
\hline $\begin{array}{l}\text { Unidentifiable } \\
\text { tube dweller }\end{array}$ & 0 & 0 & 0 & 3 & 0 & 3 & 2 & 2 & 7 & 9 & 10 & 6 & 10 & 3 & 7 & 1 & 8 & 1 & 6 \\
\hline $\begin{array}{l}\text { Unidentifiable } \\
\text { animal }\end{array}$ & 8 & 7 & 8 & 0 & 1 & 0 & 1 & 0 & 1 & 3 & 0 & 2 & 1 & 1 & 2 & 0 & 3 & 3 & 4 \\
\hline $\begin{array}{l}\text { Unknown } \\
\text { (flora or fauna) }\end{array}$ & 3 & 0 & 1 & 1 & 1 & 2 & 2 & 0 & 3 & 2 & 6 & 4 & 5 & 3 & 8 & 1 & 6 & 0 & 1 \\
\hline
\end{tabular}

were significantly different during the fall of 1999 at both Site 2 and Caspersen Beach, and during June 2000 at Caspersen Beach.

\section{Feeding selectivity}

Czekanowski's Index of Proportional Similarity showed no strong correlations between the diet of Arbacia punctulata and Lytechinus variegatus with the available flora found over different substrates (Table 6). Moderate similarity (25 to $75 \%$ ) was noted at Site 1 between the diet of A. punctulata and flora found over rubble in October 1999 and April 2000. The diet of L. variegatus was also moderately similar to flora available over sand and rubble at Caspersen Beach in June 2000. This was one of 3 collections when flora was equally abundant over sand and rubble, as indicated by the high index of similarity in the last column of Table 6 .

Comparison of the available proportions of flora in each habitat and flora consumed by sea urchins using Stauss's Feeding Selectivity Index indicates that Arbacia punctulata and Lytechinus variegatus tend to graze in a random manner on most species of flora.

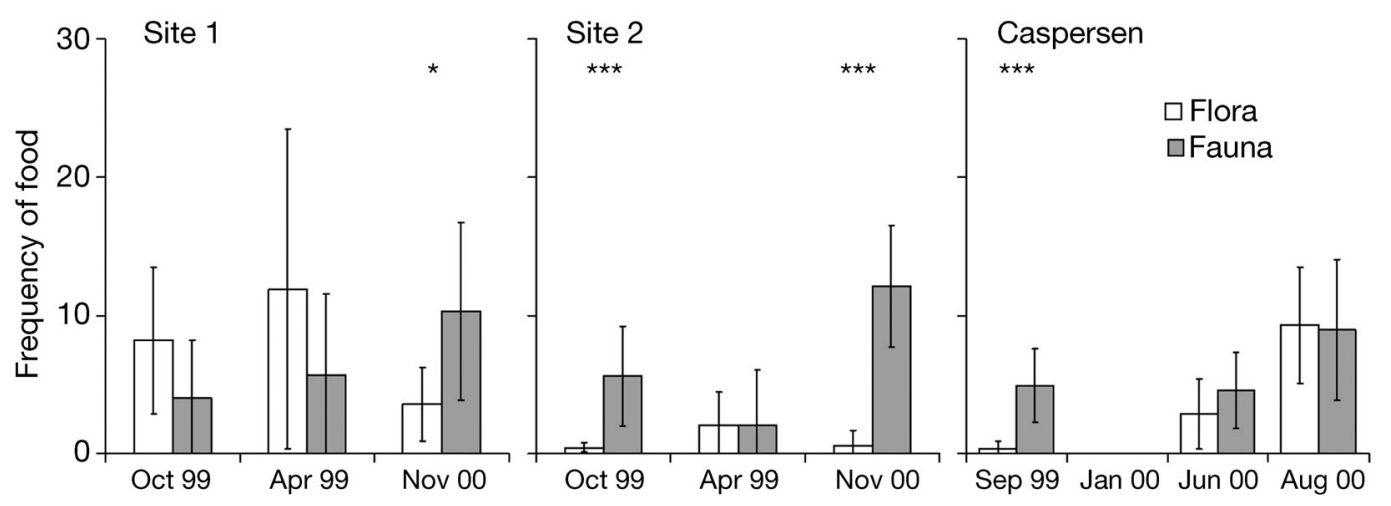

Fig. 2. Arbacia punctulata Frequency of ingested flora and fauna by point counts. Error bars represent $95 \%$ confidence interval. $(\mathrm{n}=10)$. ${ }^{*} \mathrm{p}<0.05$, $\star \star * \mathrm{p}<0.001$
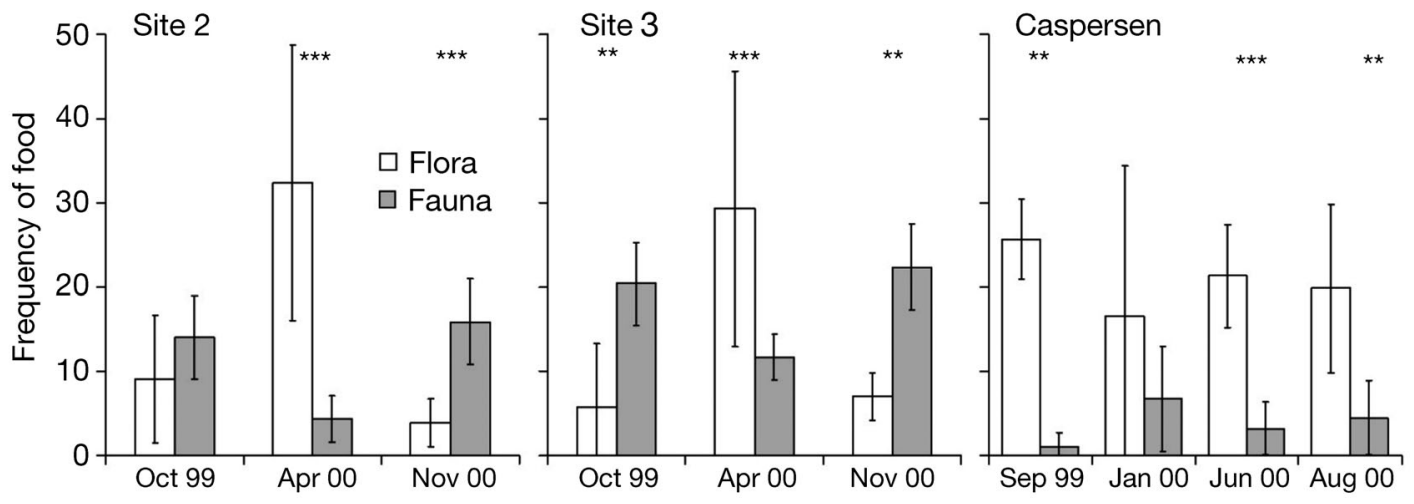

Fig. 3. Lytechinus variegates. Frequency of ingested flora and fauna by point counts. Error bars represent $95 \%$ confidence interval. ( $\mathrm{n}=10$ on all collection dates except in August 2000, where $\mathrm{n}=7$ ). ${ }^{\star *} \mathrm{p}<0.01,{ }^{\star \star *} \mathrm{p}<0.001$ 
Table 5. Arbacia punctulata and Lytechinus variegatus. Contingency coefficient values of the diets (flora and fauna) of (A) each species between different sites and dates and (B) between coexisting species at Site 2 and Caspersen Beach. Possible values range from 0.00 (identical diets) to 1.00 (dissimilar diets). Underlined values indicate statistically significant comparisons $(\mathrm{p}<0.05)$

\begin{tabular}{|c|c|c|c|c|c|c|c|c|c|c|c|}
\hline \multirow{2}{*}{\multicolumn{2}{|c|}{ (A) Arbacia punctulata }} & \multicolumn{3}{|c|}{ Site 1} & \multicolumn{3}{|c|}{ Site 2} & \multicolumn{3}{|c|}{ Caspersen } & \\
\hline & & Oct 99 & Apr 00 & Nov 00 & Oct 99 & Apr 00 & Nov 00 & Sep 99 & Jun 00 & Aug 00 & \\
\hline \multirow[t]{3}{*}{ Site 1} & Oct 99 & 0.00 & $\underline{0.54}$ & 0.53 & $\underline{0.55}$ & $\underline{0.66}$ & $\underline{0.64}$ & $\underline{0.66}$ & $\underline{0.71}$ & $\underline{0.70}$ & \\
\hline & Apr 00 & - & 0.00 & 0.51 & 0.55 & $\underline{0.60}$ & 0.61 & $\underline{0.69}$ & $\underline{0.66}$ & $\underline{0.67}$ & \\
\hline & Nov 00 & - & - & 0.00 & 0.53 & $\overline{0.65}$ & $\overline{0.60}$ & $\underline{0.66}$ & $\underline{0.71}$ & $\overline{0.68}$ & \\
\hline \multirow[t]{3}{*}{ Site 2} & Oct 99 & - & - & - & 0.00 & 0.64 & 0.53 & 0.57 & 0.64 & 0.66 & \\
\hline & Apr 00 & - & - & - & - & $\overline{0.00}$ & 0.62 & 0.71 & $\overline{0.60}$ & $\overline{0.65}$ & \\
\hline & Nov 00 & - & - & - & - & - & 0.00 & 0.55 & 0.61 & 0.61 & \\
\hline \multirow[t]{3}{*}{ Caspersen } & Sep 99 & - & - & - & - & - & - & 0.00 & 0.57 & 0.54 & \\
\hline & Jun 00 & - & - & - & - & - & - & - & 0.00 & 0.41 & \\
\hline & Aug 00 & - & - & - & - & - & - & - & - & 0.00 & \\
\hline \multirow{2}{*}{\multicolumn{2}{|c|}{ Lytechinus variegatus }} & \multicolumn{3}{|c|}{ Site 3} & \multicolumn{3}{|c|}{ Site 2} & \multicolumn{3}{|c|}{ Caspersen } & \\
\hline & & Oct 99 & Apr 00 & Nov 00 & Oct 99 & Apr 00 & Nov 00 & Sep 99 & Jan 00 & Jun 00 & Aug 00 \\
\hline \multirow[t]{3}{*}{ Site 3} & Oct 99 & 0.00 & $\underline{0.59}$ & 0.48 & $\underline{0.54}$ & $\underline{0.67}$ & 0.51 & $\underline{0.67}$ & $\underline{0.65}$ & $\underline{0.66}$ & $\underline{0.64}$ \\
\hline & Apr 00 & - & $\overline{0.00}$ & 0.52 & $\underline{0.61}$ & $\overline{0.49}$ & $\underline{0.59}$ & $\underline{0.68}$ & $\overline{0.65}$ & $\underline{0.64}$ & $\overline{0.66}$ \\
\hline & Nov 00 & - & - & 0.00 & $\overline{0.51}$ & $\underline{0.61}$ & $\overline{0.46}$ & $\underline{0.68}$ & $\underline{0.65}$ & $\underline{0.66}$ & $\underline{0.67}$ \\
\hline \multirow[t]{3}{*}{ Site 2} & Oct 99 & - & - & - & 0.00 & $\underline{0.63}$ & 0.40 & $\underline{0.68}$ & $\underline{0.66}$ & $\underline{0.65}$ & $\underline{0.66}$ \\
\hline & Apr 00 & - & - & - & - & $\overline{0.00}$ & $\underline{0.63}$ & $\underline{0.67}$ & $\underline{0.64}$ & $\underline{\overline{0.64}}$ & $\underline{0.65}$ \\
\hline & Nov 00 & - & - & - & - & - & $\overline{0.00}$ & $\underline{0.68}$ & $\underline{\overline{0.66}}$ & $\underline{0.67}$ & $\underline{\overline{0.66}}$ \\
\hline \multirow[t]{4}{*}{ Caspersen } & Sep 99 & - & - & - & - & - & - & 0.00 & $\underline{0.62}$ & $\underline{0.62}$ & $\underline{0.60}$ \\
\hline & Jan 00 & - & - & - & - & - & - & - & 0.00 & $\underline{0.57}$ & $\underline{0.61}$ \\
\hline & Jun 00 & - & - & - & - & - & - & - & - & 0.00 & 0.53 \\
\hline & Aug 00 & - & - & - & - & - & - & - & - & - & 0.00 \\
\hline \multirow{4}{*}{\multicolumn{2}{|c|}{$\begin{array}{l}\text { (B) Arbacia punctulata vs. } \\
\text { Lytechinus variegatus }\end{array}$}} & \multicolumn{3}{|c|}{ Site 2} & & & & \multicolumn{3}{|c|}{ Caspersen } & \\
\hline & & & Oct 99 & $\underline{0.60}$ & & & & Sep & & $\underline{0.60}$ & \\
\hline & & & Apr 00 & $\overline{0.57}$ & & & & Jun & & $\underline{0.59}$ & \\
\hline & & & Nov 00 & 0.56 & & & & Aug & & $\overline{0.53}$ & \\
\hline
\end{tabular}

Table 6. Arbacia punctulata and Lytechinus variegatus. Czekanowski's Index of Proportional Similarity of the diet (macroflora only) of sea urchins to different microhabitats within offshore and nearshore sites. Possible values range from 0.00 (dissimilar) to 1.00, which indicates identical proportions of algae species in the gut of the sea urchins and in the environment. Algal availability over sand and rubble substrates is also compared in the last column. n/a: comparison is not applicable because sea urchins were not present or data is unavailable

\begin{tabular}{|c|c|c|c|c|c|c|c|c|}
\hline Site & $\begin{array}{c}\text { Date } \\
\text { (mo/d/yr) }\end{array}$ & $\begin{array}{c}\text { A. punctulata } \\
\times \\
\text { Rubble }\end{array}$ & $\begin{array}{c}\text { A. punctulata } \\
\times \\
\text { Sand }\end{array}$ & $\begin{array}{c}\text { A. punctulata } \\
\times \\
\text { Rubble } \\
\text { and sand }\end{array}$ & $\begin{array}{c}\text { L. variegatus } \\
\times \\
\text { Rubble }\end{array}$ & $\begin{array}{c}\text { L. variegatus } \\
\times \\
\text { Sand }\end{array}$ & $\begin{array}{c}\text { L. variegatus } \\
\times \\
\text { Rubble } \\
\text { and sand }\end{array}$ & $\begin{array}{c}\text { Sand } \\
\times \\
\text { Rubble }\end{array}$ \\
\hline Site 1 & $\begin{array}{r}10 / 2 / 99 \\
4 / 8 / 00 \\
11 / 12 / 00\end{array}$ & $\begin{array}{l}0.58 \\
0.51 \\
0.37\end{array}$ & $\begin{array}{l}0.28 \\
0.25 \\
0.37\end{array}$ & $\begin{array}{l}0.58 \\
0.50 \\
0.37\end{array}$ & $\begin{array}{l}\mathrm{n} / \mathrm{a} \\
\mathrm{n} / \mathrm{a} \\
\mathrm{n} / \mathrm{a}\end{array}$ & $\begin{array}{l}\mathrm{n} / \mathrm{a} \\
\mathrm{n} / \mathrm{a} \\
\mathrm{n} / \mathrm{a}\end{array}$ & $\begin{array}{l}\mathrm{n} / \mathrm{a} \\
\mathrm{n} / \mathrm{a} \\
\mathrm{n} / \mathrm{a}\end{array}$ & $\begin{array}{l}0.26 \\
0.25 \\
0.34\end{array}$ \\
\hline Site 2 & $\begin{array}{r}10 / 2 / 99 \\
4 / 8 / 00 \\
11 / 12 / 00\end{array}$ & $\begin{array}{l}0.24 \\
0.05 \\
0.00\end{array}$ & $\begin{array}{l}0.24 \\
0.01 \\
0.00\end{array}$ & $\begin{array}{l}0.24 \\
0.04 \\
0.00\end{array}$ & $\begin{array}{l}0.25 \\
0.05 \\
0.00\end{array}$ & $\begin{array}{l}0.13 \\
0.01 \\
0.00\end{array}$ & $\begin{array}{l}0.23 \\
0.04 \\
0.00\end{array}$ & $\begin{array}{l}0.88 \\
0.02 \\
0.00\end{array}$ \\
\hline Site 3 & $\begin{array}{r}10 / 2 / 99 \\
4 / 8 / 00 \\
11 / 12 / 00\end{array}$ & $\begin{array}{l}\mathrm{n} / \mathrm{a} \\
\mathrm{n} / \mathrm{a} \\
\mathrm{n} / \mathrm{a}\end{array}$ & $\begin{array}{l}\mathrm{n} / \mathrm{a} \\
\mathrm{n} / \mathrm{a} \\
\mathrm{n} / \mathrm{a}\end{array}$ & $\begin{array}{l}\mathrm{n} / \mathrm{a} \\
\mathrm{n} / \mathrm{a} \\
\mathrm{n} / \mathrm{a}\end{array}$ & $\begin{array}{l}0.24 \\
\mathrm{n} / \mathrm{a} \\
0.34\end{array}$ & $\begin{array}{l}0.00 \\
0.31 \\
0.34\end{array}$ & $\begin{array}{l}0.00 \\
\mathrm{n} / \mathrm{a} \\
0.34\end{array}$ & $\begin{array}{l}0.00 \\
\mathrm{n} / \mathrm{a} \\
0.81\end{array}$ \\
\hline $\begin{array}{l}\text { Caspersen } \\
\text { Beach }\end{array}$ & $\begin{array}{l}9 / 29 / 99 \\
1 / 17 / 00 \\
6 / 15 / 00 \\
8 / 23 / 00\end{array}$ & $\begin{array}{l}0.00 \\
\mathrm{n} / \mathrm{a} \\
0.27 \\
0.24\end{array}$ & $\begin{array}{l}0.00 \\
\mathrm{n} / \mathrm{a} \\
0.27 \\
0.33\end{array}$ & $\begin{array}{l}0.00 \\
\mathrm{n} / \mathrm{a} \\
0.27 \\
0.36\end{array}$ & $\begin{array}{l}0.02 \\
0.37 \\
0.47 \\
0.25\end{array}$ & $\begin{array}{l}0.00 \\
0.00 \\
0.56 \\
0.27\end{array}$ & $\begin{array}{l}0.00 \\
0.00 \\
0.50 \\
0.31\end{array}$ & $\begin{array}{l}0.00 \\
0.00 \\
0.74 \\
0.22\end{array}$ \\
\hline
\end{tabular}


Macroflora consumed with moderate to strong index values are listed in Tables 7 \& 8. Both sea urchins exhibited a moderate to strong avoidance for species of Halimeda, Dictyota, and Helminthocladia. Moderate avoidance was noted for Laurencia spp. and Caulerpa spp. by A. punctulata, while the preference of $L$. variegatus for these species fluctuated with date. Both species of sea urchins showed a preference for Botryocladia occidentalis and Agardhiella spp. on some dates and avoided these species on other occasions. Neither sea urchin strongly favored any species of algae.

\section{DISCUSSION}

A primary factor controlling diets of benthic herbivores is availability of food. With the exception of offshore Site 1, a highly variable diversity of flora occurred at both nearshore and offshore sites (Cobb \& Lawrence 2003). Although Site 1 was only $15 \mathrm{~km}$ from Sites 2 and 3, which were only $193 \mathrm{~m}$ apart, floral community composition varied greatly (Cobb \& Lawrence 2003).

Given the low abundance of macroalgal availability at most sites and limited distance chemoreception of sea urchins (De Ridder \& Lawrence 1982), the likelihood of frequent encounters of attached algae by sea urchins should be low. With limited availability of attached fleshy macroalgae and plants in the Gulf (Cobb \& Lawrence 2003), consumers may be forced to rely on other sources of food such as encrusting coralline algae, drift algae, and animals.

High repletion indices for Arbacia punctulata at Site 1 indicate high consumption, suggesting food is not a limited resource and reducing the probability of intraspecific competition for food. Lower repletion indices support the conclusion that Site 2 is nutritionally poor in comparison to Site 1. Caspersen Beach also consistently had low repletion indices. Caspersen Beach may be food limited but possibly not to the same extent as Site 2.

Lytechinus variegatus at Sites 2 and 3 may rely heavily on food other than attached algae. This is indicated by peaks in the repletion index during April 2000 which coincided with the spring bloom of drifting Cladophora spp., although total floral biomass was not significantly higher during the spring than in the fall. High repletion indices during January 2000 at Caspersen Beach are inconsistent with previous behavioral patterns of $L$. variegatus. $L$. variegatus normally decreases feeding activity in the winter when food is limited and temperatures are low (Klinger \& Lawrence 1984). However, the repletion index does not indicate when feeding occurred, as gut passage time increases when feeding decreases. Dietary preference is often difficult to determine in the field due to variability in the supply of food, which can result

Table 7. Arbacia punctulata Feeding selectivity. Values range from -1.00 (avoidance) to 1.00 (preference) with 0.00 indicating random feeding. Only foods with values greater than 0.24 or less than -0.24 are included. All other food found in the gut was ingested randomly on all dates. Dashes indicate days when a species of algae was not present in either the gut or in the environment

\begin{tabular}{|c|c|c|c|c|c|c|c|c|c|}
\hline \multirow{2}{*}{$\begin{array}{l}\text { Site } \\
\text { Species }\end{array}$} & \multicolumn{3}{|c|}{ Date $(\mathrm{mo} / \mathrm{d} / \mathrm{yr})$} & \multicolumn{3}{|c|}{ Date $(\mathrm{mo} / \mathrm{d} / \mathrm{yr})$} & \multicolumn{3}{|c|}{ Date $(\mathrm{mo} / \mathrm{d} / \mathrm{yr})$} \\
\hline & Rubble & Sand & $\begin{array}{l}\text { Rubble } \\
\text { \& sand }\end{array}$ & Rubble & Sand & $\begin{array}{l}\text { Rubble } \\
\& \text { sand }\end{array}$ & Rubble & Sand & $\begin{array}{l}\text { Rubble } \\
\text { \& sand }\end{array}$ \\
\hline Site 1 & \multicolumn{3}{|c|}{$-10 / 2 / 99 \square$} & \multicolumn{3}{|c|}{$4 / 8 / 00$} & \multicolumn{3}{|c|}{$-11 / 12 / 00$} \\
\hline Caulerpa sp. & -0.03 & -0.49 & -0.04 & 0.00 & -0.03 & -0.01 & - & - & - \\
\hline Halimeda discoidea & -0.10 & - & -0.10 & -0.02 & -0.67 & -0.10 & -0.01 & - & 0.00 \\
\hline Botryocladia occidentalis & 0.21 & 0.29 & 0.22 & -0.05 & 0.01 & -0.04 & -0.01 & 0.00 & -0.01 \\
\hline Laurencia spp. & -0.15 & 0.22 & -0.14 & -0.31 & 0.11 & -0.26 & -0.53 & -0.29 & -0.48 \\
\hline \multicolumn{4}{|l|}{ Site 2} & \multicolumn{3}{|c|}{$4 / 8 / 00$} & \multicolumn{3}{|c|}{$-11 / 12 / 00$} \\
\hline Caulerpa prolifera & - & - & - & - & - & - & - & -1.00 & 0.00 \\
\hline Halimeda discoidea & - & -0.12 & -0.02 & -0.38 & - & -0.34 & -0.17 & - & -0.17 \\
\hline Dictyota spp. & -0.64 & -0.64 & -0.64 & 0.03 & 0.03 & 0.03 & 0.00 & - & 0.00 \\
\hline Agardhiella subulata & - & - & - & -0.56 & - & -0.50 & - & - & - \\
\hline Botryocladia occidentalis & - & - & - & - & - & - & -0.83 & - & -0.83 \\
\hline Gelidium spp. & 0.10 & 0.10 & 0.10 & 0.26 & 0.26 & 0.26 & 0.25 & 0.25 & 0.25 \\
\hline Helminthocladia sp. & - & - & - & -0.01 & -0.99 & -0.12 & - & - & - \\
\hline Lobophora variegata & 0.10 & 0.10 & 0.10 & 0.05 & 0.05 & 0.05 & 0.38 & 0.38 & 0.38 \\
\hline \multicolumn{4}{|l|}{ Caspersen Beach } & \multicolumn{3}{|c|}{$6 / 15 / 00$} & \multicolumn{3}{|c|}{$8 / 23 / 00$} \\
\hline Sargassum filipendula & -0.62 & - & -0.62 & - & - & - & -0.01 & - & -0.01 \\
\hline Agardhiella spp. & 0.44 & 0.44 & 0.44 & - & - & - & 0.04 & 0.04 & 0.04 \\
\hline Chondria spp. & - & - & - & - & - & - & -0.38 & - & -0.22 \\
\hline Family Rhodomelaceae (4) & - & - & - & 0.44 & 0.45 & 0.44 & 0.21 & 0.21 & 0.21 \\
\hline Gracilaria spp. & -0.36 & - & -0.36 & - & -0.26 & -0.02 & 0.12 & -0.52 & -0.16 \\
\hline Hypnea spp. & 0.11 & 0.11 & 0.11 & -0.72 & -0.47 & -0.70 & -0.08 & 0.01 & -0.04 \\
\hline
\end{tabular}


Table 8. Lytechinus variegatus. Feeding selectivity. Values range from -1.00 (avoidance) to 1.00 (preference) with 0.00 indicating random feeding. Only foods with values greater than 0.24 or less than -0.24 are included. All other food found in the gut was ingested randomly on all dates . Dashes indicate days when a species of algae was not present in either the gut or in the environment

\begin{tabular}{|c|c|c|c|c|c|c|c|c|c|c|c|c|}
\hline \multirow{2}{*}{$\begin{array}{l}\text { Site } \\
\text { Species }\end{array}$} & \multicolumn{3}{|c|}{ Date $(\mathrm{mo} / \mathrm{d} / \mathrm{yr})$} & \multicolumn{3}{|c|}{ Date $(\mathrm{mo} / \mathrm{d} / \mathrm{yr})$} & \multicolumn{3}{|c|}{ Date $(\mathrm{mo} / \mathrm{d} / \mathrm{yr})$} & \multicolumn{3}{|c|}{ Date $(\mathrm{mo} / \mathrm{d} / \mathrm{yr})$} \\
\hline & Rubble & Sand & $\begin{array}{l}\text { Rubble } \\
\& \text { sand }\end{array}$ & Rubble & Sand & $\begin{array}{l}\text { Rubble } \\
\text { \& sand }\end{array}$ & Rubble & Sand & $\begin{array}{l}\text { Rubble } \\
\text { \& sand }\end{array}$ & Rubble & Sand & $\begin{array}{l}\text { Rubble } \\
\& \text { sand }\end{array}$ \\
\hline Site 2 & - & 10/2/99 & - & $\longrightarrow$ & $4 / 8 / 00$ & - & -1 & $1 / 12 / 0$ & $0-$ & & & \\
\hline Caulerpa spp. & - & - & - & 0.01 & 0.01 & 0.01 & - & -1.00 & 0.00 & & & \\
\hline Halimeda discoidea & - & -0.12 & -0.02 & -0.38 & - & -0.34 & -0.17 & - & -0.17 & & & \\
\hline Dictyota spp. & -0.75 & -0.75 & -0.75 & 0.00 & 0.00 & 0.00 & 0.04 & 0.04 & 0.04 & & & \\
\hline Agardhiella subulata & - & - & - & -0.56 & - & -0.50 & - & - & - & & & \\
\hline $\begin{array}{l}\text { Botryocladia } \\
\text { occidentalis }\end{array}$ & - & - & - & 0.04 & 0.04 & 0.04 & -0.83 & - & -0.83 & & & \\
\hline Helminthocladia sp. & - & - & - & -0.01 & -0.99 & -0.12 & - & - & - & & & \\
\hline Laurencia spp. & 0.46 & 0.46 & 0.46 & 0.07 & 0.07 & 0.07 & 0.46 & 0.46 & 0.46 & & & \\
\hline Site 3 & -1 & $10 / 2 / 99$ & - & - & $4 / 8 / 00$ & - & -1 & $1 / 12 / 0$ & - & & & \\
\hline Caulerpa spp. & 0.36 & 0.36 & 0.36 & - & -0.68 & - & - & -0.19 & -0.04 & & & \\
\hline $\begin{array}{l}\text { Halimeda discoidea } \\
\text { Botryocladia }\end{array}$ & - & - & - & - & - & - & -0.54 & - & -0.51 & & & \\
\hline occidentalis & -0.30 & - & -0.30 & - & - & - & - & - & - & & & \\
\hline Caspersen Beach & 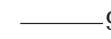 & 9/29/99 & 9 & - & $1 / 17 / 00$ & 0 & 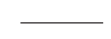 & $6 / 15 / 00$ & - & - & $3 / 23 / 00$ & 0 \\
\hline Sargassum sp(p). & -0.62 & - & -0.62 & 0.02 & 0.02 & 0.02 & - & - & - & -0.01 & - & -0.01 \\
\hline $\begin{array}{l}\text { Agardhiella spp. } \\
\text { Botryocladia }\end{array}$ & 0.12 & 0.12 & 0.12 & 0.23 & 0.58 & 0.23 & 0.14 & 0.14 & 0.14 & 0.08 & 0.08 & 0.08 \\
\hline occidentalis & 0.61 & 0.63 & 0.61 & 0.01 & 0.01 & 0.01 & - & - & - & -0.06 & -0.01 & -0.04 \\
\hline Chondria $\mathrm{sp}(\mathrm{p})$. & - & - & - & -0.53 & 0.01 & -0.53 & - & - & - & -0.38 & - & -0.22 \\
\hline Gracilaria spp. & -0.36 & - & -0.36 & 0.14 & 0.14 & 0.14 & 0.09 & -0.17 & 0.07 & 0.06 & -0.58 & -0.21 \\
\hline Hурпеа spp. & 0.07 & 0.07 & 0.07 & 0.05 & 0.05 & 0.05 & -0.52 & -0.27 & -0.50 & -0.06 & 0.03 & -0.02 \\
\hline
\end{tabular}

in a change in food preference over time as resources become limited (Boudouresque \& Verlaque 2001). While some sea urchins are strictly herbivorous or carnivorous, the sea urchins in this study are clearly omnivorous and change their diet based on resource availability.

Arbacia punctulata consumed equal proportions of flora and fauna only during periods when attached and drifting macroflora were very abundant, such as October 1999 and April 2000 at Site 1. During seasons of moderate to low floral biomass, for example November 2000 at Site 1 or fall collections at Site 2 (Cobb \& Lawrence 2003), A. punctulata consumed animals almost exclusively. Strauss's Selectivity Index indicates that $A$. punctulata consumed almost all species of algae in a random manner. This implies that $A$. punctulata is a general omnivore when algal abundance is high and is normally carnivorous when algal availability is moderate to low, as is often the case on the Florida gulf coast shelf.

In contrast to Arbacia punctulata, Lytechinus variegatus exhibits a stronger preference for algal and plant matter than animals. At Sites 2 and 3, L. variegatus primarily consumed animals when macroflora abundance was low. However, during April 2000, when combined macroflora biomass over rubble and sand was moderately high and drift algae were very abundant (Cobb \&
Lawrence 2003), L. variegatus consumed significantly more algal matter. Macroflora abundance at Caspersen Beach was low to moderate on all dates (Cobb \& Lawrence 2003); however, L. variegatus consumed much more algal matter than animals, except in January 2000. Like $A$. punctulata, random feeding was more common than selection at all sites. This is consistent with laboratory experiments, suggesting that L. variegatus detects food only within $8 \mathrm{~cm}$ (Klinger \& Lawrence 1984). Random feeding has been documented in seagrass beds based primarily on macroflora availability (Vadas et al. 1982).

Because Arbacia punctulata and Lytechinus variegatus primarily fed in a random manner and showed no strong preferences for any species of algae, neither sea urchin ate large quantities of particular species. Both sea urchins often avoided the calcareous species Halimeda discoidea and 2 fleshy species Dictyota spp. and Helminthocladia sp. As Sargassum sp. was qualitatively observed to be in high abundance at Site 1, it can be assumed that $A$. punctulata also avoided consumption of this species. All of these species contain large amounts of secondary metabolites that deter herbivory (Hay \& Fenical 1988). Laurencia spp. and Caulerpa spp., 2 genera that contain anti-herbivory compounds, were often avoided during periods of high algal availability, and consumed in greater quantities by L. varie- 
gatus during fall collections when floral biomass was low. The Mediterranean sea urchin Paracentrotus lividus consumes less palatable species such as Codium fragile and encrusting algae when resources are limited (Boudouresque \& Verlaque 2001).

At Caspersen Beach Arbacia punctulata did not consume large amounts of Sargassum filipendula, Gracilaria spp., or Hypnea spp. In laboratory experiments species of Sargassum and Dictyota are avoided by $A$. punctulata, presumably due to secondary metabolites (Hay et al. 1986, Cronin \& Hay 1996, Cronin et al. 1997), while species of Gracilaria and Hypnea are considered preferred foods (Hay \& Fenical 1988). One explanation is that these species occur more often as drift algae at Caspersen Beach and may not be an available to $A$. punctulata because it lacks suckered podia, which facilitates the capture of drifting food (De Ridder \& Lawrence 1982). However, the arbaciod Tetrapygus niger captures drift algae (Rodríguez 2003). Lytechinus variegatus frequently fed on many species of drift algae with the exception of Sargassum sp. at Caspersen Beach. Drift Rhodophyta were still consumed in lower proportions than found in the environment. Unlike A. punctulata, L. variegatus possesses podia with suckers (Flammang 1996) and captures drift algae (J. Cobb unpubl. data). Although drift algae were collected in habitat sampling, it is highly probable that its biomass was underestimated. Feeding on drift algae may explain how $L$. variegatus consumed greater quantities of algae at Caspersen Beach, and during spring collections at offshore sites, than appeared available from floral biomass estimates.

In addition to macroflora, both species consumed a diverse range of animals, many of which contained indigestible structures. Ascidians of the family Didemnidae, which possess stellate spicules of calcium carbonate, often epiphytized Laurencia sp. and were frequently consumed by Arbacia punctulata. Other animals primarily included sessile species such as encrusting bryozoans, barnacles, hydrozoans, sponges, and tube dwelling organisms. Errant invertebrates such as mollusks and crustaceans were consumed less frequently. Consumption of animals with large quantities of inorganic material suggests that sea urchins may feed indiscriminately on animals as well as algae. However, sampling may be biased with respect to softbodied organisms that would be rapidly digested and difficult for researchers to identify.

Restricted feeding by Arbacia punctulata to a particular substrate could not be detected by Czekanowski's index, except when algal availability was exceptionally high over rubble at Site 1 . In this case $A$. punctulata exhibited a moderate preference for algae over rubble. No other correlation with substrate was detected at Site 2, Site 3, or Caspersen Beach for A. punctulata or Lytechinus variegatus. The probability of detecting a true pattern between the feeding of sea urchins and a particular substrate by a similarity index is greatly diminished by low abundance of each algal species and the tendency of $A$. punctulata to consume large quantities of animals. In addition, available flora over rubble and sand were quite similar on some dates. This observation is attributed to extremely low diversity and biomass of macroflora. Although the reliability of Czekanowski's index can be questioned when flora are sparse, A. punctulata was always observed on rubble outcroppings and $L$. variegatus was primarily found on sand. Many sessile animals consumed, such as sponges, tunicates, and hydroids, were only observed on rubble outcroppings. This implies that $A$. punctulata and $L$. variegatus fed on rubble at least during periods when algal availability was low. Some species of algae consumed by L. variegatus, such as Caulerpa, were only observed over sand or shell hash. Based on qualitative observations it is likely that $L$. variegatus at least feeds on both types of substrate.

All collections in this study were conducted during the day, although feeding preferences over different substrates may change during the night. Laboratory experiments by Hay et al. (1986) demonstrated that chemotaxis and feeding preferences of Arbacia punctulata show diurnal rhythms. Like the tropical seaurchin Diadema setosum (Lawrence \& Hughes-Games 1972), A. punctulata and Lytechinus variegatus feed more during the night (J. Cobb unpubl. data).

As Arbacia punctulata tends to consume large quantities of animals while Lytechinus variegatus primarily consumes plants and algae, it is not surprising that very little overlap in the diet of these species was detected using the contingency coefficient at either Caspersen Beach or Site 2. Interspecific competition is therefore unlikely, except under conditions of extremely low flora availability, such as those observed at Site 2 in 2000, when both species may feed solely on animals. This is also supported by comparison of diets between sampling sites that contain 1 species of sea urchin and sites with both species. Comparisons between offshore sites indicate moderate overlap between the diets of sea urchins of the same species which solely dominate an area (A. punctulata at Site 1 or $L$. variegatus at Site 3 ) and sites where species coexist (Site 2). If interspecific competition for food existed, we would expect a shift in diet of both species at Site 2 because of the presence of a competitor. Differences in diet of offshore populations and nearshore populations are most likely attributed to differences in diversity of algae available at these sites, although this can not be verified without proper replicates. Macroalgae diversity was more similar between offshore locations compared to Caspersen Beach. 
In summary, diets of Arbacia punctulata and Lytechinus variegatus do not overlap significantly at sites along the Florida gulf coast shelf. Both species feed randomly on most species of algae when available. Competition for food is unlikely as A. punctulata primarily consumes animal foods while $L$. variegatus primarily consumes algae and plants. Shallow sites less than $12 \mathrm{~m}$ in depth appear to exhibit more variability in community composition with season. When floral biomass decreases at these locations, competition for food may increase as both species consume larger quantities of animals. Drift algae may also play an important role in the diet of $L$. variegatus at sites of low floral biomass. Tokeshi (1999) stated that a small overlap in resource utilization is likely due to (1) competition over evolutionary and/or contemporary time, (2) independent evolutionary diversification in niche characteristics, or (3) phylogenetic distance between species resulting in little overlap in niche characters, including pattern of resource use. We do not know whether the progenitors of Arbacia punctulata and Lytechinus variegatus competed. Our observations indicate they do not compete now as they occupy distinct microhabitats except in heterogeneous situations. This seems to be the result of their distinct morphological characteristics.

Acknowledgements. We would like to thank C. Dawes, B. Cowell and J. Garey for their advice and assistance, the captains and crews of the RV 'Bellows' and RV 'Suncoaster,' the Florida Institute of Oceanography for ship-time support on the 'Bellows' and 'Suncoaster', American Academy of Underwater Sciences divers of the University of South Florida Department of Biology for assistance with collections, and $\mathrm{J}$. Vincente for assistance processing samples. This study was supported in part by Florida Sea Grant R/LR-A-21 to J.M.L.

\section{LITERATURE CITED}

Beddingfield SD, McClintock JB (1999) Food resource utilization in the sea urchin Lytechinus variegatus in contrasting shallow-water microhabitats of Saint Joseph Bay, Florida. Gulf Mex Sci 1:27-34

Benedetti-Cecchi L, Cinelli F (1995) Habitat heterogeneity, sea urchins grazing and the distribution of algae in littoral rock pools on the west coast of Italy (western Mediterranean). Mar Ecol Prog Ser 126:203-212

Benedetti-Cecchi L, Bulleri F, Cinelli F (1998) Density dependent foraging of sea urchins in shallow subtidal reefs on the west coast of Italy (western Mediterranean). Mar Ecol Prog Ser 163:203-211

Boudouresque CF, Verlaque M (2001) The ecology of Paracentrotus lividus. In: Lawrence JM (ed) Edible sea urchins: biology and ecology. Elsevier Science Press, Amsterdam, p $177-216$

Bulleri F, Benedetti-Cecchi L, Cinelli F (1999) Grazing by the sea urchins Arbacia lixula L. and Paracentrotus lividus Lam. in the northwest Mediterranean. J Exp Mar Biol Ecol 241:81-95

Bulleri F, Bertocci I, Micheli F (2002) Interplay of encrusting coralline algae and sea urchins in maintaining alternative habitats. Mar Ecol Prog Ser 243:101-109

Cobb JC (2002) A dietary comparison of offshore and nearshore populations of the co-occurring sea urchins Lytechinus variegatus and Arbacia punctulata (Echinodermata) along the central Florida Gulf shelf. MS thesis, University of South Florida, Tampa, FL

Cobb JC, Lawrence JM (2003) Seasonal and spatial variation in algal composition and biomass on the central Florida Gulf-coast shelf. Gulf Mex Sci 21:192-201

Couto ECG (1998) Hábito alimentar do ourico-do-mar Lytechinus variegatus (Lamarck) (Echinodermata: Echinoidea) e distribucão de macroalgas no Saco do Limoeiro (Ilha do Mel, PR). 22 Congresso Brasileiro do Zoologica Program, Abstract

Cronin G, Hay ME (1996) Susceptibility to herbivores depends on recent history of both the plant and animal. Ecology 77:1531-1543

Cronin G, Paul VJ, Hay ME, Fenical W (1997) Are tropical herbivores more resistant than temperate herbivores to seaweed chemical defenses? Diterpenoid metabolites from Dictyota acutiloba as feeding deterrents for tropical versus temperate fishes and urchins. J Chem Ecol 23: 289-302

Dawes CJ (1974) Marine algae of the west coast of Florida. University of Miami Press, Coral Gables, FL

De Ridder C, Lawrence JM (1982) Food and feeding mechanisms: Echinoidea. In: Jangoux M, Lawrence JM (eds) Echinoderm nutrition. AA Balkema, Rotterdam, p 499-519

Flammang P (1996) Adhesion in echinoderms. Echinoderm Stud 5:1-60

Frantzis A, Berthon JF, Maggiore F (1988) Relations trophiques entre les oursins Arbacia lixula et Paracentrotus lividus (Echinoidea Regularia) et le phytobenthos infralittoral superficial dans la Baie de Port-Cros (Var, France). Sci Rep Port-Cros Natl Park, Fr 14:81-140 (English Abstract)

Fraser CM (1944) Hydroids of the Atlantic Coast of North America. University of Toronto Press, Toronto

Greenway M (1995) Trophic relationships of macrofauna within a Jamaican seagrass meadow and the role of the echinoid Lytechinus variegatus (Lamarck). Bull Mar Sci 56:719-736

Harvey EB (1956) The American Arbacia and other sea urchins. Princeton University Press, Princeton, NJ

Hay ME, Fenical W (1988) Marine plant-herbivore interactions: The ecology of chemical defense. Annu Rev Ecol Syst 19:111-145

Hay ME, Lee RR Jr., Guieb RA, Bennett MM (1986) Food preference and chemotaxis in the sea urchin Arbacia punctulata (Lamarck) Philippi. J Exp Mar Biol Ecol 96:147-153

Hendler G, Miller JE, Pawson DL, Kier PM (1995) Sea stars, sea urchins, and allies. Smithsonian Institution Press, Washington, DC

Hill SK, Lawrence JM (2003) Habitats and characteristics of the sea urchins Lytechinus variegatus and Arbacia punctulata (Echinodermata) on the Florida gulf-coast shelf. PSZN I: Mar Ecol 24:15-30

Jones RS (1968) A suggested method for quantifying gut contents in herbivorous fishes. Micronesica 4:369-371

Karlson R (1975) The effects of predation by the sea urchin, Arbacia punctulata on a marine epibenthic community. PhD dissertation, Duke University, Durham, NC

Keller BD (1983) Coexistence of sea urchins in seagrass meadows: an experimental analysis of competition and predation. Ecology 64:1581-1598 
Klinger TS, Lawrence JM (1984) Food and movement of Lytechinus variegatus (Lamarck) (Echinodermata: Echinoidea). Abstract. Am Zool 24:29A

Lawrence JM, Hughes-Games L (1972) The diurnal rhythm of feeding and passage of food through the gut of Diadema setosum (Echinodermata: Echinoidea). Isr J Zoo 21:13-16

Lowe EF, Lawrence JM (1976) Absorption efficiencies of Lytechinus variegatus (Lamarck) (Echinodermata: Echinoidea) for selected marine plants. J Exp Mar Biol Ecol 21: $223-234$

Moore HB, Jutare T, Bauer JC, Jones JA (1963) The biology of Lytechinus variegatus. Bull Mar Sci Gulf Caribb 13:23-53

Parker GH (1932) On certain feeding habits of the sea-urchin Arbacia. Am Nat 66:95-96

Penchaszadeh P, Lawrence JM (1999) Arbacia dufresnei (Echinodermata: Echinoidea): a carnivore in Argentinian waters. In: Candia Carnevali MD, Bonasoro F (eds) Echinoderm research 1998. Balkema, Rotterdam, p 526-530

Plough HH (1978) Sea squirts of the Atlantic Continental Shelf from Maine to Texas. John Hopkins University Press, Baltimore, MD

Régis MB (1980) Étude des possibilities d'élevage des oursins réguliers en function de la valeur de certains indices physiologiques. Oceanol Acta 3:7-15 (English Abstract)

Rodríguez SR (2003) Consumption of drift kelp by intertidal populations of the sea urchin Tetrapygus niger on the central Chilean coast: possible consequences at different ecological levels. Mar Ecol Prog Ser 251:141-151

Ruppert EE, Fox RS (1988) Seashore animals of the Southeast. University of South Carolina Press, Columbia, SC

Shier DE (1964) Marine Bryozoa from northwest Florida. Bull Mar Sci Gulf Caribb 14:603-746

Sterrer W (1986) Marine fauna and flora of Bermuda. A sys-

Editorial responsibility: Otto Kinne (Editor-in-Chief), Oldendorf/Luhe, Germany tematic guide to the identification of marine organisms. John Wiley \& Sons, New York

Strauss RE (1979) Reliability estimates for Ivlev's electivity index, the forage ratio, and a proposed linear index of food selection. Trans Am Fish Soc 108:344-352

Tegner MJ (2001) The ecology of Strongylocentrotus franciscanus and Strongylocentrotus purpuratus. In: Lawrence JM (ed) Edible sea urchins: biology and ecology. Elsevier Science Press, Amsterdam, p 307-331

Tokeshi M (1999) Species coexistence: ecological and evolutionary perspectives. Blackwell Science, Oxford

Vadas RL (1985) Herbivory. In: Littler MM, Littler DS (eds) Handbook of physiological methods. Cambridge University Press, Cambridge, p 537-545

Vadas RL, Fenchel T, Ogden JC (1982) Ecological studies on the sea urchin, Lytechinus variegatus, and the algalseagrass communities of the Miskito Cays, Nicaragua. Aquat Bot 14:109-125

Van Name WG (1945) The North and South American ascidians. Bull Am Mus Nat Hist 84. American Museum of Natural History, New York

Vásquez JA (2001) Ecology of Loxechinus albus. In: Lawrence JM (ed) Edible sea urchins: biology and ecology. Elsevier Science Press, Amsterdam, p 161-175

Verlaque M (1984) Biology of juveniles of the herbivorous sea urchin, Paracentrotus lividus (Lamarck): selectivity of grazing and impact on rocky algal communities in Corsica (Mediterranean, France). Bot Mar 27:401-424

Verlaque M, Nédelec H (1983) Biologie de Paracentrotus lividus (Lamarck) sur substrat rocheux en Corse (Méditerranée, France): Alimentation des adultes. Vie Milieu 33: 191-201 (English Abstract)

Wynne MJ (1986) A checklist of benthic marine algae of the tropical and subtropical western Atlantic. Can J Bot 64: $2239-2281$

Submitted: September 30, 2003; Accepted: November 11, 2004 Proofs received from author(s): June 6, 2005 